\title{
Uterine leiomyomas: correlation between histologic composition and stiffness via magnetic resonance elastography - a Pilot Study
}

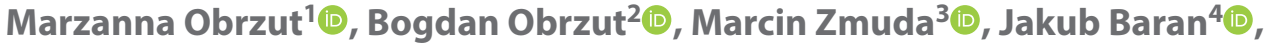 \\ Marian Cholewa ${ }^{1}$, Richard L. Ehman ${ }^{5}{ }^{\circledR}$, Dorota Darmochwal-Kolarz²® \\ ${ }^{1}$ Department of Biophysics, Faculty of Mathematics and Natural Sciences, University of Rzeszow, Poland \\ ${ }^{2}$ Department of Obstetrics and Gynecology, Institute of Medical Sciences, Medical College, University of Rzeszow, Poland \\ ${ }^{3}$ Department of Clinical Pathomorphology, Provincial Clinical Hospital No 2 Rzeszow, Poland \\ ${ }^{4}$ Institute of Nuclear Physics Polish Academy of Sciences, Cracow, Poland \\ ${ }^{5}$ Department of Radiology, Mayo Clinic, USA
}

\begin{abstract}
Objectives: To evaluate magnetic resonance elastography as a tool for characterizing uterine leimyomas.

Material and methods: At total of 12 women with symptomatic leiomyomas diagnosed in physical and ultrasound examinations were enrolled in this pilot study. Before surgery, all patients underwent magnetic resonance elastography of the uterus using a 1.5 T MR whole-body scanner (Optima, GE Healthcare, Milwaukee, WI, USA). Surgical specimens were forwarded for histological examination. The findings were allocated into 3 categories depending on the percentage content of connective tissue: below $15 \%$, from 15 to $30 \%$ and more than $30 \%$. The median stiffness of leiomyomas for each of the group was calculated. The U-Mann Whitney test was used for statistical analysis.

Results: The stiffness of the leiomyomas ranged between 3.7-6.9 kPa (median value $4.9 \mathrm{kPa}$ ). The concentration of extracellular components in the leiomyomas did not exceed $40 \%$. An increasing trend of the stiffness with the growing percentage of extracellular component was observed. Stiffness of the leiomyomas obtained by MRE varies depending on microscopic composition.

Conclusions: The value of stiffness shows a trend of increasing with the percentage of extracellular component of the leiomyoma. Further studies are required to assess the usefulness of MRE in diagnostics of uterine leiomyomas.

Key words: leiomyoma; magnetic resonance elastography; histologic composition
\end{abstract}

Ginekologia Polska 2020; 91, 7: 373-378

\section{INTRODUCTION}

Uterine leiomyomas (fibroids) are the most common benign gynaecological tumours, which affect up to $70 \%$ of women of a reproductive age [1-3]. They develop as a result of the transformation of myometrial smooth muscle, fibroblasts and the formation of a dysfunctional extracellular matrix $[4,5]$. The majority of fibroids remain asymptomatic, 20-40\% of women with leiomyomas report significant clinical symptoms (e.g. abnormal uterine bleeding, dysmenorrhea, pelvic pain, infertility, and recurrent pregnancy loss) that require effective gynaecological intervention $[2,6,7]$.

The management of uterine fibromas varies significantly depending on the patient's age, symptoms, and reproductive plans. For women who wish to preserve their fertility and avoid surgery, there are numerous medical therapy options available [8]. For symptomatic women after medical treatment failures, surgical procedures are proposed [9]. Strategies for surgical treatment depend on the patient and fibroid characteristics and include myomectomy (in women who want to preserve their uterus or desire future pregnancy) or hysterectomy (for women after a completed childbearing) [9]. Currently, both procedures are mostly performed via laparoscopy with the usage of a power morcellator. Laparoscopic power morcellation is related with a risk of spreading undiagnosed neoplastic tissue within the abdominal cavity, which may significantly worsen the patient's prognosis [10-12]. The true prevalence of uterine sarcomas in presumed fibroids is not exactly known and 
vary from $0.09 \%$ to $0,8 \%$ depending on the study [13-17]. Also, the medical management of presumed symptomatic leiomyoma, which is found to be sarcoma, may have a negative impact on the outcome due to a delay in appropriate therapy. Despite the fact that some patients' characteristics and abnormal features of uterine tumors on a US or MRI may be suggestive for the presence of leiomyosarcoma, a reliable differential diagnosis is still very difficult $[13,18-20]$. Hence, there is an urgent need for finding a new tool that could improve the characterization of uterine masses. In this context, interesting possibilities are offered in recently implemented magnetic resonance elastography (MRE). Malignant transformation often markedly alters the mechanical properties of soft tissue [21,22], which could be potentially used in the pre-treatment differentiating leiomyosarcoma from the common leiomyoma. On the other hand, elastographic patterns of the leiomyoma, reflecting a relative concentration of cellular and extracellular components, may be helpful in predicting a response to non- excisional therapies such as uterine artery embolization and magnetic resonance-guided focused ultrasound [23]. Both circumstances require knowledge of the typical elastographic features of leiomyomas.

\section{Objectives}

Until now, only two studies have been published on the feasibility of MRE for uterine leiomyomas, without regard to their microscopic composition [23,24]. The aim of this study was to evaluate the correlation between the elasticity of leiomyomas obtained by MRE and their relative concentration of cellular and extracellular components.

\section{MATERIAL AND METHODS}

\section{Patient population}

This pilot study included 12 patients with symptomatic leiomyomas, who underwent surgical treatment at the Clinical Department of Obstetrics and Gynaecology of Provincial Clinical Hospital No 2, Rzeszow, Poland between September 2016 and February 2017.

Preoperative diagnosis was based on vaginal speculum examination, bimanual examination and the transvaginal ultrasonography of the uterus. Before surgery, all patients underwent laboratory tests, electrocardiography, anesthesiology consultation and magnetic resonance elastography of the uterus. Written consent was obtained from each of the patients before the study commenced. The research protocol was approved by the Bioethics Committee of the University of Rzeszow, Poland (Reg. No. 19/04/2016).

\section{Magnetic Resonance Elastography}

A 1.5T MR whole-body scanner (Optima, GE Healthcare, Milwaukee, WI, USA) was used to perform the MRE scans. The
MRE system, includes special acquisition and processing software, as well as hardware consisting of an active and passive driver. The passive driver is a small plastic drum-like device that is placed against the body to transmit mechanical vibrations into tissue. In this study, the passive driver was placed over the uterus in the lower abdomen. The passive driver is connected via flexible tubing to the active driver unit which is located outside the MRI room. The active driver unit generates pressure pulses (at $60 \mathrm{~Hz}$ frequency in this study) that are conducted to the passive driver via the flexible tubing.

MRE acquisition was performed using a 16-channel abdominal phase array coil. Shear wave imaging was conducted using a modified $2 \mathrm{D}$ gradient-recalled echo-based pulse sequence with the following parameters: matrix size: $256 \times 256$, slice thickness: $10 \mathrm{~mm}$; TR: $33 \mathrm{~ms}$; TE: $20 \mathrm{~ms}$; flip angle: $30 \mathrm{deg}$. The resulting wave images were then automatically processed by the scanner using a manufacturer-provided $2 \mathrm{D}$ direct inversion algorithm to generate quantitative images depicting tissue stiffness (elastograms) [25-27].

Region of interest (ROI) were drawn manually on the largest leiomyoma, avoiding non-fibroid tissue, using corresponding $\mathrm{T} 2$ images as a guide. From the ROI, mean stiffness $(\mathrm{kPa})$ and standard deviation, were reported.

\section{Microscopic evaluation}

Surgical specimens were forwarded for histological examination. To visualise the collagenous connective tissue fibers in tissue sections and differentiate them from smooth muscle fibers, Trichrome Stain (Connective Tissue Stain) was applied. In this process staining muscle fibers were dyed red, collagen fibers were stained blue and nuclei stained black/blue (Fig. 1). Due to lack of similar publications and other scientific data, the findings were arbitrarily allocated into 3 categories depending on the percentage content of connective tissue (below $15 \%$, from 15 to $30 \%$, more than $30 \%$ ).

\section{Statistical analysis}

Statistical analysis was performed using Python libraries. The U-Mann Whitney tests were applied. A p value of lower than 0.05 was considered statistically significant.

\section{RESULTS}

A greater number of patients were premenopausal than not. Only one patient was postmenopausal. Their age ranged between $26-61$ years (median value 40.5 years). The median body mass index (BMI) was $22.45 \mathrm{~kg} / \mathrm{m}^{2}$. The parity status and type of surgery are presented in Table 1.

In all patients, the preoperative diagnosis leiomyoma was confirmed histologically. The diameter of the largest leiomyoma ranged between 40-109 mm (median diameter $68.5 \mathrm{~mm}$ ). The median value of leiomyoma stiffness obtained 
in our series was $4.9 \mathrm{kPa}$ (range, 3.7-6.9) The concentration of extracellular components in the leiomyomas and their stiffness in specific patients are presented in Table 2.

The biggest subgroup was made up of leiomyomas with contents of fibrous tissue up to 15 percent. The median stiffness in this series was $4.46 \mathrm{kPa}$. Three patients had leiomyomas with a concentration of extracellular components between 15 and $30 \%$ and median stiffness of $5.78 \mathrm{kPa}$, respectively. More than $30 \%$ content of fibrous tissue was found in 2 patients. The median stiffness of those leiomyo-

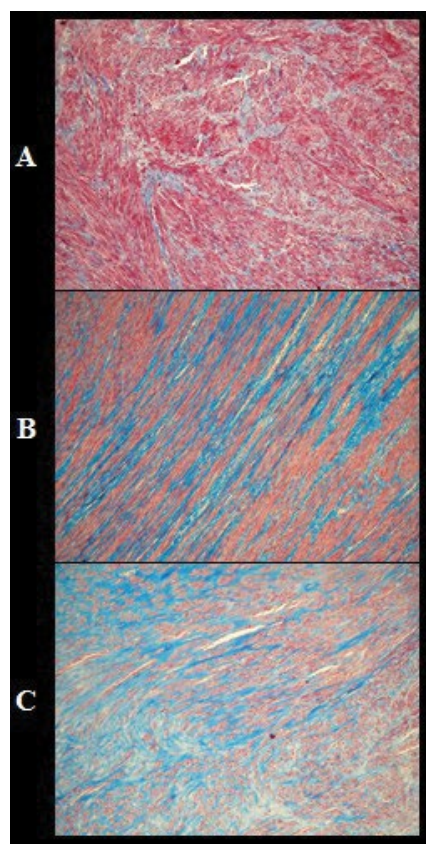

Figure 1. Microscopic pictures of leiomyomas with different histologic composition: fibrous tissue content up to $15 \%$ (A), between $15 \%$ and $30 \%(B)$, and above $30 \%(C)$ in trichrome stain mas was $6.15 \mathrm{kPa}$. Example ROl's drawn on elastograms for three patients are shown in Figure 2.

Figure 3 depicts a box plot with the mean stiffness measurements among groups with different extracellular component. Despite the low number of samples, the increasing trend of the stiffness with the growing percentage of extracellular component is clearly observable. The p value for the stiffness difference between lesions with extracellular component of $<15 \%$ and $>30 \%$ was $p=0.05$. Other results were not statistically significant.

\section{DISCUSSION}

Magnetic resonance elastography is a versatile MRI-based technique, using propagating shear waves to

\begin{tabular}{|c|c|c|c|c|}
\hline Patient & $\begin{array}{l}\text { Diameter } \\
\text { of the } \\
\text { leiomyoma } \\
\text { [mm] }\end{array}$ & $\begin{array}{l}\text { Extracellular } \\
\text { component } \\
{[\%]}\end{array}$ & $\begin{array}{l}\text { Stiffness } \\
\text { of the } \\
\text { leiomyoma }\end{array}$ & $\begin{array}{l}\text { SD of } \\
\text { leiomyomas' } \\
\text { stiffness }\end{array}$ \\
\hline 1 & 57 & $<15$ & 6.18 & 0.68 \\
\hline 2 & 41 & $<15$ & 3.7 & 1.2 \\
\hline 3 & 109 & $>30$ & 6.9 & 2.8 \\
\hline 4 & 40 & $<15$ & 4.5 & 1.5 \\
\hline 5 & 56 & $<15$ & 4.2 & 1.02 \\
\hline 6 & 62 & $<15$ & 4.0 & 1.2 \\
\hline 7 & 70 & $<15$ & 4.9 & 1.3 \\
\hline 8 & 75 & $<15$ & 4.97 & 1.08 \\
\hline 9 & 67 & $15-30$ & 4.68 & 1.06 \\
\hline 10 & 93 & $>30$ & 5.39 & 0.90 \\
\hline 11 & 82 & $15-30$ & 5.8 & 2.2 \\
\hline 12 & 80 & $15-30$ & 5.9 & 1.6 \\
\hline
\end{tabular}

\begin{tabular}{|c|c|c|c|c|c|}
\hline Patient & Age & Gravidity/Parity & Hormonal status & BMI & Type of surgery \\
\hline 1 & 49 & $2 / 2$ & premenopausal & 20.42 & LASH/BS \\
\hline 2 & 33 & 0 & premenopausal & 21.48 & laparotomy/myomectomy \\
\hline 3 & 32 & 0 & premenopausal & 24.77 & laparotomy/myomectomy \\
\hline 4 & 28 & $1 / 1$ & premenopausal & 19.16 & laparoscopy/myomectomy \\
\hline 5 & 38 & $3 / 2$ & premenopausal & 23.42 & LASH/BS \\
\hline 6 & 31 & $2 / 2$ & premenopausal & 26.17 & LASH/BS \\
\hline 7 & 43 & $3 / 1$ & premenopausal & 20.76 & LASH/BS \\
\hline 8 & 48 & $2 / 2$ & premenopausal & 21.09 & TLH/BS \\
\hline 9 & 26 & 0 & premenopausal & 27.02 & laparotomy/myomectomy \\
\hline 10 & 46 & 0 & premenopausal & 21.05 & laparotomy/myomectomy \\
\hline 11 & 49 & $2 / 2$ & premenopausal & 25.10 & TLH/BS \\
\hline 12 & 61 & $3 / 1$ & postmenopausal & 27.24 & TAH/BSO \\
\hline
\end{tabular}

LASH — laparoscopic supracervical hysterectomy; TLH — total laparoscopic hysterectomy; TAH — total abdominal hysterectomy; BS — bilateral salpingectomy; BSO - bilateral salpingooophorectom 


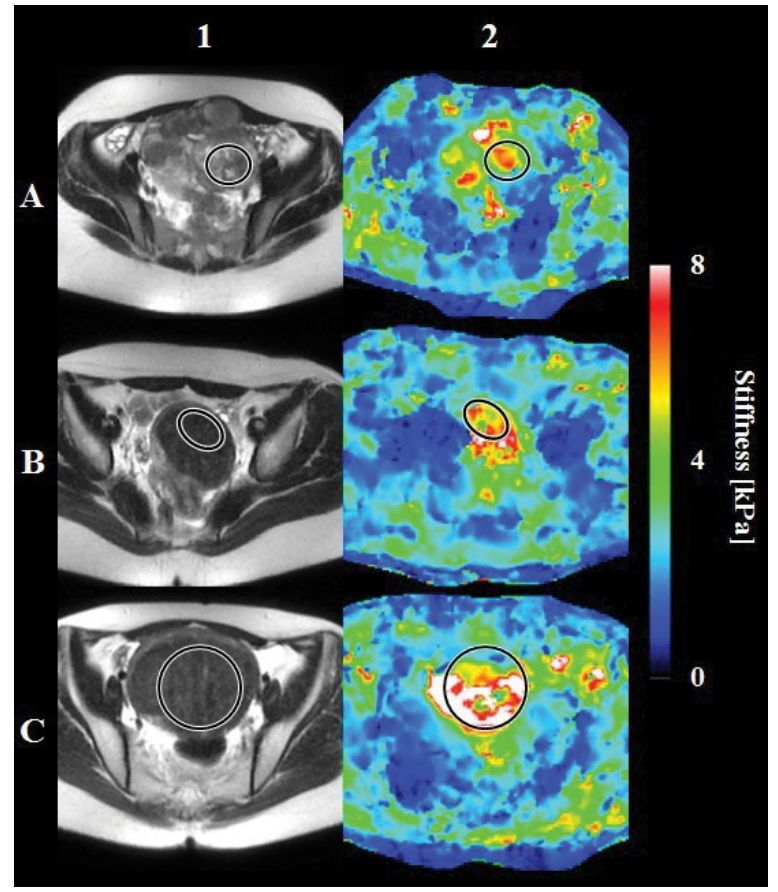

Figure 2. T2w MR images (column 1) and comparison of elastograms (column 2) for patient 6 (row A), 9 (row B) and 3 (row C). The mean stiffness was $3.95 \mathrm{kPa}, 4.68 \mathrm{kPa}$ and $6.9 \mathrm{kPa}$ for the above mentioned patients, respectively

assess the mechanical properties of tissue [28]. The most established and documented clinical application of MRE is the evaluation of chronic liver disease [29-33]. In recent years, MRE has also been evaluated in characterization of focal liver lesions [22,34], and for assessing the spleen, pancreas, kidneys and prostate [35-39]. Experiences with MRE in the field of gynaecology are quite limited. There are only a few publications dealing with the stiffness of a normal uterus [40] and leiomyomas [23, 24]. None of the research groups investigated the stiffness of leiomyomas in correlation with their histologic composition. In this context, our research provides data to fill this gap.

The present study confirms the findings of Stewart et al. [23], that magnetic resonance elastography is a feasible technique for the assessment of mechanical properties of uterine leiomyomas. The median value of leiomyoma stiffness obtained in our series $(4.9 \mathrm{kPa})$ was comparable to that of above cited study.

The stiffness of the leiomyoma is markedly higher than the stiffness of both the normal corpus and cervix uteri, which were evaluated by Jiang et al. using 3D MRE technique [40]. Compared to other pathologic lesions, the elasticity of leiomyomas exceeds those of benign liver tumors (mean $2.7 \mathrm{kPa}$, range $1.6-3.2 \mathrm{kPa}$ ), but it is similar to the elasticity of fibrotic liver disease (mean $5.9 \mathrm{kPa}$, range 3.1-12.2 kPa) [22].

The content of the extracellular component in leiomyomas from our series did not exceed $40 \%$. The concentration

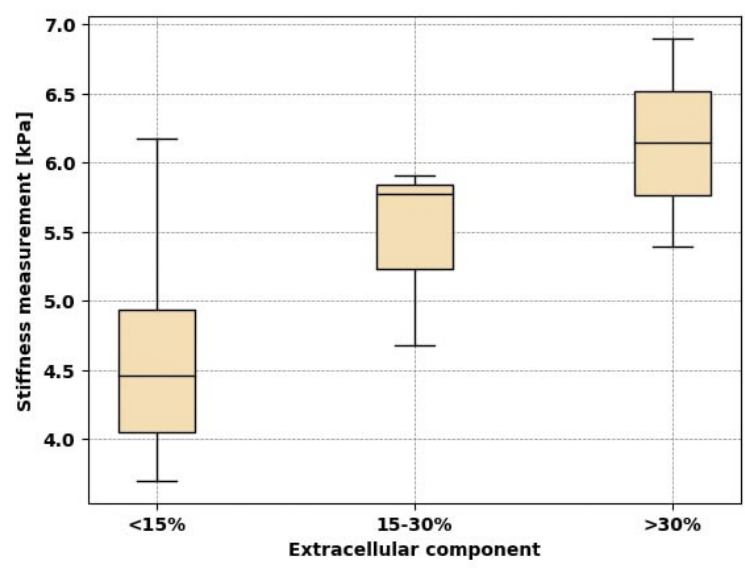

Figure 3. Box plot depicting variance in shear stiffness measurements for all groups of patients

of collagen fibres correlated with the value of stiffness of the leiomyoma (Fig. 3). Leiomyoma stiffness, as an increasing trend, is observable with the increased extracellular component; however, statistical analysis only approached demonstrating a significant difference between the groups with the highest and lowest collagen concentration. The relatively large scatter of stiffness values in the group of lowest extracellular component is probably due to the inhomogeneity of leiomyomas.

To the best of our knowledge, this is the first study investigating the correlation between the elasticity of leiomyomas obtained by MRE and their relative concentration of cellular and extracellular components. The presented observations can be potentially helpful in preoperative differentiating common leiomyomas from their malign counterparts. Because uterine sarcomas tend to be more cellular, they may be able to be distinguished from the leiomyomas based on the altered mechanical properties. This expectation is also related to the need for preoperative distinguishing between common leiomyoma and cellular leiomyoma, as they may have premalignant potential [41,42]. Our series included neither leiomyosarcomas, nor cellular leiomyoma, so this hypothesis is purely theoretical. However, it seems to be justified based on similar observations regarding breast and liver tumors [21, 22, 34].

The limitation of the presented paper is the small size of the study group, and its one-institutional character. Multi-centre research with a larger patient series will be required to gain appropriate statistical power to objectively assess the usefulness of MRE in evaluating the microscopic composition of leiomyomas. Besides, in our study two-dimensional MRE have been used, which is limited in estimating the true mechanical property of tissue, especially in small lesions. It was the reason for our patient selection and qualifying only those with middle-size and larger leiomyoma. In the follow- 
ing investigations a 3D-MRE should be used to overcome this limitation.

\section{CONCLUSIONS}

In conclusion, this study demonstrates that stiffness of the leiomyomas obtained by MRE varies depending on their histologic composition. The value of stiffness shows a trend of increasing with growing percentage of extracellular component of the leiomyoma. Further investigations with a larger patient group and 3D-MRE are needed to assess the usefulness of MRE in evaluating leiomyomas and defining its possible clinical application.

\section{Acknowledgments}

The study was performed within the project "Centre for Innovative Research in Medical and Natural Sciences" realized by the University of Rzeszów, co-financed within the Regional Operational Program for the Podkarpackie Province for the years 2007-2013 (contract number UDA-RPPK.01.03.00-18004/12-00)

\section{REFERENCES}

1. Lumsden MA. Modern management of fibroids. Obstetrics, Gynaecology \& Reproductive Medicine. 2013; 23(3): 65-70, doi: 10.1016/j. ogrm.2013.02.001

2. Marsh EE, Ekpo GE, Cardozo ER, et al. Racial differences in fibroid prevalence and ultrasound findings in asymptomatic young women (18-30 years old): a pilot study. Fertil Steril. 2013; 99(7): 1951-1957, doi: 10.1016/j.fertnstert.2013.02.017, indexed in Pubmed: 23498888.

3. Segars JH, Parrott EC, Nagel JD, et al. Proceedings from the Third National Institutes of Health International Congress on Advances in Uterine Leiomyoma Research: comprehensive review, conference summary and future recommendations. Hum Reprod Update. 2014; 20(3): 309-333, doi: 10.1093/humupd/dmt058, indexed in Pubmed: 24401287.

4. Chegini N. Proinflammatory and profibrotic mediators: principal effectors of leiomyoma development as a fibrotic disorder. Semin Reprod Med. 2010; 28(3): 180-203, doi: 10.1055/s-0030-1251476, indexed in Pubmed: 20414842

5. Ryan GL, Syrop CH, Van Voorhis BJ. Role, epidemiology, and natural history of benign uterine mass lesions. Clin Obstet Gynecol. 2005; 48(2): 312-324, doi: 10.1097/01.grf.0000159538.27221.8c, indexed in Pubmed: 15805789.

6. Downes E, Sikirica V, Gilabert-Estelles J, et al. The burden of uterine fibroids in five European countries. Eur J Obstet Gynecol Reprod Biol. 2010; 152(1): 96-102, doi: 10.1016/j.ejogrb.2010.05.012, indexed in Pubmed: 20598796.

7. Pritts EA, Parker WH, Olive DL. Fibroids and infertility: an updated systematic review of the evidence. Fertil Steril. 2009; 91(4): 1215-1223, doi: 10.1016/j.fertnstert.2008.01.051, indexed in Pubmed: 18339376.

8. Kashani BN, Centini G, Morelli SS, et al. Role of Medical Management for Uterine Leiomyomas. Best Pract Res Clin Obstet Gynaecol. 2016; 34: 85-103, doi: 10.1016/j.bpobgyn.2015.11.016, indexed in Pubmed: 26796059.

9. Thubert $T$, Foulot $H$, Vinchant $M$, et al. Surgical treatment: Myomectomy and hysterectomy; Endoscopy: A major advancement. Best Pract Res Clin Obstet Gynaecol. 2016; 34: 104-121, doi: 10.1016/j.bpobgyn.2015.11.021, indexed in Pubmed: 27400649.

10. Perri T, Korach J, Sadetzki S, et al. Uterine leiomyosarcoma: does the primary surgical procedure matter? Int J Gynecol Cancer. 2009; 19(2): 257-260, doi: 10.1111/IGC.0b013e31819a1f8f, indexed in Pubmed: 19396005.

11. Park JY, Park SK, Kim DY, et al. The impact of tumor morcellation during surgery on the prognosis of patients with apparently early uterine leiomyosarcoma. Gynecol Oncol. 2011; 122(2): 255-259, doi: 10.1016/j. ygyno.2011.04.021, indexed in Pubmed: 21565389.
12. George S, Barysauskas C, Serrano C, et al. Retrospective cohort study evaluating the impact of intraperitoneal morcellation on outcomes of localized uterine leiomyosarcoma. Cancer. 2014; 120(20): 3154-3158, doi: 10.1002/cncr.28844, indexed in Pubmed: 24923260.

13. Leibsohn S, d'Ablaing G, Mishell D, et al. Leiomyosarcoma in a series of hysterectomies performed for presumed uterine leiomyomas. American Journal of Obstetrics and Gynecology. 1990; 162(4): 968-976, doi: 10.1016/0002-9378(90)91298-q.

14. Uterine Leiomyoma. SpringerReference., doi: 10.1007/springerreference 177792.

15. Rowland M, Lesnock J, Edwards R, et al. Occult uterine cancer in patients undergoing laparoscopic hysterectomy with morcellation: Implications for surveillance for disease recurrence and outcomes. Gynecologic Oncology. 2013; 130(1): e77, doi: 10.1016/j.ygyno.2013.04.243.

16. Ehdaivand S, Simon RA, Sung CJ, et al. Incidental gynecologic neoplasms in morcellated uterine specimens: a case series with follow-up. Hum Pathol. 2014; 45(11): 2311-2317, doi: 10.1016/j.humpath.2014.07.018, indexed in Pubmed: 25257577.

17. Seidman MA, Oduyebo T, Muto MG, et al. Peritoneal dissemination complicating morcellation of uterine mesenchymal neoplasms. PLoS One. 2012; 7(11): e50058, doi: 10.1371/journal.pone.0050058, indexed in Pubmed: 23189178.

18. Goto A, Takeuchi S, Sugimura K, et al. Usefulness of Gd-DTPA contrast-enhanced dynamic MRI and serum determination of LDH and its isozymes in the differential diagnosis of leiomyosarcoma from degenerated leiomyoma of the uterus. Int J Gynecol Cancer. 2002; 12(4): 354-361, doi: 10.1046/j.1525-1438.2002.01086.x, indexed in Pubmed: 12144683.

19. Tanaka YO, Nishida M, Tsunoda $H$, et al. Smooth muscle tumors of uncertain malignant potential and leiomyosarcomas of the uterus: MR findings. J Magn Reson Imaging. 2004; 20(6): 998-1007, doi: 10.1002/jmri.20207, indexed in Pubmed: 15558559.

20. Testa $A C$, Di Legge $A$, Bonatti $M$, et al. Imaging techniques for evaluation of uterine myomas. Best Pract Res Clin Obstet Gynaecol. 2016; 34:37-53, doi: 10.1016/j.bpobgyn.2015.11.014, indexed in Pubmed: 26803557.

21. Krouskop TA, WheelerTM, Kallel F, et al. Elastic moduli of breast and prostate tissues under compression. Ultrason Imaging. 1998; 20(4): 260-274, doi: 10.1177/016173469802000403, indexed in Pubmed: 10197347.

22. Venkatesh SK, Yin M, Glockner JF, et al. MR elastography of liver tumors: preliminary results. AJR Am J Roentgenol. 2008; 190(6): 1534-1540, doi: 10.2214/AJR.07.3123, indexed in Pubmed: 18492904.

23. Stewart EA, Taran FA, Chen J, et al. Magnetic resonance elastography of uterine leiomyomas: a feasibility study. Fertil Steril. 2011; 95(1): 281-284, doi: 10.1016/j.fertnstert.2010.06.004, indexed in Pubmed: 20633880.

24. Jondal DE, Wang J, Chen J, et al. Uterine fibroids: correlations between MRI appearance and stiffness via magnetic resonance elastography. Abdom Radiol (NY). 2018; 43(6): 1456-1463, doi: 10.1007/s00261-0171314-1, indexed in Pubmed: 28952003.

25. Manduca A, Oliphant TE, Dresner MA, et al. Magnetic resonance elastography: non-invasive mapping of tissue elasticity. Med Image Anal. 2001; 5(4): 237-254, doi: 10.1016/s1361-8415(00)00039-6, indexed in Pubmed: 11731304

26. Oliphant TE, Manduca A, Ehman RL, et al. Complex-valued stiffness reconstruction for magnetic resonance elastography by algebraic inversion of the differential equation. Magn Reson Med. 2001; 45(2): 299-310, doi: 10.1002/1522-2594(200102)45:2<299::aid-mrm1039>3. 0.co;2-o, indexed in Pubmed: 11180438.

27. Manduca A, Lake DS, Kruse SA, et al. Spatio-temporal directional filtering for improved inversion of MR elastography images. Med Image Anal. 2003; 7(4): 465-473, doi: 10.1016/s1361-8415(03)00038-0, indexed in Pubmed: 14561551.

28. Venkatesh SK, Ehman RL. Magnetic resonance elastography of abdomen Abdom Imaging. 2015; 40(4): 745-759, doi: 10.1007/s00261-014-0315-6, indexed in Pubmed: 25488346

29. Ichikawa S, Motosugi U, Ichikawa T, et al. Magnetic resonance elastography for staging liver fibrosis in chronic hepatitis C. Magn Reson Med Sci. 2012; 11(4): 291-297, doi: 10.2463/mrms.11.291, indexed in Pubmed: 23269016.

30. Hennedige TP, Wang G, Leung FP, et al. Magnetic resonance elastography for the detection and staging of liver fibrosis in chronic hepatitis B. Eur Radiol. 2014; 24(1): 70-78, doi: 10.1007/s00330-013-2978-8, indexed in Pubmed: 23928932

31. Huwart L, Sempoux C, Vicaut E, et al. Liver fibrosis: noninvasive assessment with MR elastography versus aspartate aminotransferase-to-platelet ratio index. Radiology. 2007; 245(2): 458-466, doi: 10.1148/radiol.2452061673, indexed in Pubmed: 17940304 
32. Kim BoH, Lee JM, Lee YeJi, et al. MR elastography for noninvasive assessment of hepatic fibrosis: experience from a tertiary center in Asia. J Magn Reson Imaging. 2011;34(5): 1110-1116, doi: 10.1002/jmri.22723, indexed in Pubmed: 21932355.

33. Lee Yeji, Lee JM, Lee JE, et al. MR elastography for noninvasive assessment of hepatic fibrosis: reproducibility of the examination and reproducibility and repeatability of the liver stiffness value measurement. J Magn Reson Imaging. 2014;39(2):326-331, doi: 10.1002/jmri.24147, indexed in Pubmed: 23589232.

34. Garteiser P,Doblas S, Daire JL, et al. MRelastography of liver tumours: value of viscoelastic properties for tumour characterisation. Eur Radiol. 2012; 22(10): 2169-2177, doi: 10.1007/s00330-012-2474-6, indexed in Pubmed: 22572989.

35. Nedredal Gl, Yin M, McKenzie T, et al. Portal hypertension correlates with splenic stiffness as measured with MR elastography. J Magn Reson Imaging. 2011; 34(1): 79-87, doi: 10.1002/jmri.22610, indexed in Pubmed: 21608066.

36. Shi Yu, Glaser KJ, Venkatesh SK, et al. Feasibility of using 3D MR elastography to determine pancreatic stiffness in healthy volunteers. J Magn Reson Imaging. 2015; 41(2):369-375, doi: 10.1002/jmri.24572, indexed in Pubmed: 24497052

37. Rouvière $\mathrm{O}$, Souchon R, Pagnoux G, et al. Magnetic resonance elastography of the kidneys: feasibility and reproducibility in young healthy adults. J Magn Reson Imaging. 2011; 34(4): 880-886, doi: 10.1002/jmri.22670, indexed in Pubmed: 21769970.

38. Arani A, Da Rosa M, Ramsay E, et al. Incorporating endorectal MR elastography into multi-parametric MRI for prostate cancer imaging: Initial feasibility in volunteers. J Magn Reson Imaging. 2013; 38(5): 1251-1260, doi: 10.1002/jmri.24028, indexed in Pubmed: 23408516.

39. Sahebjavaher RS, Baghani $A$, Honarvar $M$, et al. Transperineal prostate MR elastography: initial in vivo results. Magn Reson Med. 2013; 69(2): 411-420, doi: 10.1002/mrm.24268, indexed in Pubmed: 22505273.

40. Jiang $X$, Asbach $P$, Streitberger KJ, et al. In vivo high-resolution magnetic resonance elastography of the uterine corpus and cervix. Eur Radiol. 2014; 24(12): 3025-3033, doi: 10.1007/s00330-014-3305-8, indexed in Pubmed: 25038856.

41. Giuntoli RL, Gostout BS, DiMarco CS, et al. Diagnostic criteria for uterine smooth muscle tumors: leiomyoma variants associated with malignant behavior. J Reprod Med. 2007; 52(11): 1001-1010, indexed in Pubmed: 18161397.

42. Taran FA, Weaver AL, Gostout BS, et al. Understanding cellular leiomyomas: a case-control study. Am J Obstet Gynecol. 2010; 203(2): 109.e1-109.e6, doi: 10.1016/j.ajog.2010.03.018, indexed in Pubmed: 20451890. 